УДК 681.7.069

\author{
И.С. Жданов ${ }^{1,2}$, Д.С. Харенко ${ }^{1,2}$, С.А. Бабин ${ }^{1,2}$ \\ ${ }^{1}$ Институт автоматики и электрометрии СО РАН, Новосибирск, Россия \\ ${ }^{2}$ Новосибирский государственный университет, Новосибирск, Россия
}

\title{
ПОЛНОСТЬЮ ВОЛОКОННЫЙ ЭРБИЕВЫЙ ЛАЗЕР С СИНХРОНИЗАЦИЕЙ МОД С ВЫСОКОЙ ЭНЕРГИЕЙ В ИМПУЛЬСЕ
}

\begin{abstract}
Работа посвящена повышению энергии импульсов, получаемых напрямую из задающего генератора в полностью волоконном эрбиевом лазере с составным кольцевым резонатором. Путем удлинения резонатора получено существенное увеличение энергии импульсов до 3,5 нДж при сохранении стабильного режима генерации сильночирпованных диссипативных солитонов (СЧДС). Смена направления оптической накачки привела к генерации шумоподобных импульсов с энергией $~ 5,5$ нДж.
\end{abstract}

Ключевые слова: лазеры ультракоротких импульсов, волоконные лазеры, эрбиевые волоконные лазеры, нелинейное вращение поляризации.

\author{
I.S. Zhdanov ${ }^{1,2}$, D.S. Kharenko ${ }^{1,2}$, S.A. Babin ${ }^{1,2}$ \\ ${ }^{1}$ Institute of Automation and Electrometry SB RAS, Novosibirsk, Russian Federation \\ ${ }^{2}$ Novosibirsk State University, Novosibirsk, Russian Federation

\section{ALL-FIBER ERBIUM-DOPED MODE-LOCKED LASER WITH HIGH PULSE ENERGY}

This article describes an energy increase of the pulses generated from all-fiber Erbium master oscillator with a ring hybrid cavity. The energy is significantly increased up to $3.5 \mathrm{~nJ}$ with a cavity lengthening with a stable highly chirped dissipative soliton (HCDS) generation regime. Pump direction change leads to a generation of noise-like pulses with $5.5 \mathrm{~nJ}$ energy.

Keywords: Ultrafast lasers, fiber lasers, Erbium fiber lasers, nonlinear polarization evolution.

В последнее время все больший интерес вызывают мощные фемтосекундные лазеры с несущей длиной волны 1550 нм, имеющие широкий круг применений: когерентная антистоксова спектроскопия комбинационного рассеяния (coherent anti-Stokes Raman spectroscopy, CARS) [1], генерация импульсов предельно короткой длительности (порядка нескольких колебаний) [2], частотная метрология [3, 4] и ге- 
нерация терагерцового излучения [5]. Все эти применения предъявляют высокие требования к источнику излучения, такие как высокая мощность, малая длительность импульса и высокая стабильность генерации.

Режим генерации сильночирпованных (с параметром чирпа >10) диссипативных солитонов (СЧДС) позволяет создать полностью волоконную схему, обладающую всеми обозначенными параметрами. Значительное увеличение энергии СЧДС в области 1 мкм было достигнуто с применением лазеров с составным волоконным резонатором [6], состоящим из стандартного одномодового волокна (SMF) и одномодового волокна с сохранением поляризации (РM SMF). Синхронизация мод такого лазера достигается за счет использования эффекта нелинейного вращения поляризации (nonlinear polarization evolution, NPE) в SMF, а формирование СЧДС - в относительно длинном участке PM SMF. Для спектральной области 1,5 мкм этот подход был впервые применен в работе [7]. В результате была получена генерация диссипативных солитонов с длительностью 165 фс после сжатия и энергией 0,93 нДж в кольцевом эрбиевом лазере с составным резонатором. Для обеспечения интегральной нормальной дисперсии, необходимой для режима СЧДС, резонатор дополнялся одномодовым волокном, компенсирующим дисперсию (dispersion compensated fiber, DCF), но не сохраняющим поляризацию. Поэтому в полученной конфигурации повышение энергии не представлялось возможным ни за счет более мощной системы накачки, ни за счет увеличения длины резонатора из-за избыточного вращения поляризации (так называемого NPE overdriving) в DCF. Режим генерации из одноимпульсного переходил в многоимпульсный. Избежать этого можно было, укоротив участок резонатора без сохранения поляризации, что также приводило к уменьшению суммарной дисперсии и негативно сказывалось на получении стабильного режима генерации СЧДС.

Данная работа посвящена изучению возможности дальнейшего повышения энергии СЧДС, генерируемых напрямую из задающего генератора, с использованием волокна с сохранением поляризации, имеющим нормальную дисперсию в области 1,5 мкм

Схема экспериментальной установки приведена на рис. 1. Исследуемый лазер имеет кольцевой резонатор, разделенный на две части. Короткая часть без сохранения поляризации длиной 3 м составлена из 
2 м волокна DCF и 1 м стандартного одномодового волокна (single mode fiber, SMF) и позволяет синхронизовать моды резонатора за счет эффекта NPE. Остальная часть резонатора состоит исключительно из волокон с сохранением поляризации, и в ней происходит формирование диссипативного солитона. В качестве активной среды используется волокно, легированное ионами $\mathrm{Er}^{3+}$ (Nufern PM EDF 7/125), накачиваемое одномодовым лазерным диодом с максимальной мощностью 550 мВт на длине волны 980 нм. Обеспечение спектральной фильтрации, необходимой для генерации диссипативных солитонов, производилось при помощи волоконного фильтра Лио (PF - polarization filter). Фильтр представлял из себя отрезок РМ-волокна, сваренного под углом $45^{\circ}$ к основным осям резонатора. Изолятор задавал направление распространения излучения и одновременно выполнял функцию анализатора для поляризационного фильтра. Ширина фильтра варьировалась от 26 до 55 нм путем изменения длины вставки, а точное положение максимума пропускания подбиралось путем ее нагрева [8]. Для увеличения длины резонатора использовалось рамановское волокно c сохранением поляризации (PM Raman Fiber, OFS), обладающее большой нормальной дисперсией $\beta_{2}=26 \mathrm{nc}^{2} /$ км на длине волны 1550 нм, что важно для обеспечения режима генерации СЧДС. Также данное волокно характеризуется эффективной площадью моды $18,7 \pm 1,5$ мкм $^{2}$ и большим коэффициентом ВКР (рамановского) усиления $-2,5(\text { Вт·км })^{-1}$.

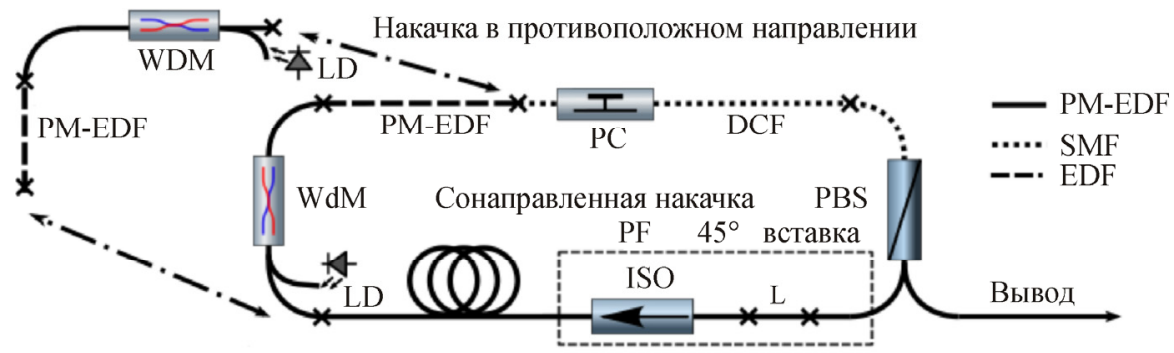

РМ Рамановское волокно

Рис. 1. Схема лазера в конфигурации с сонаправленной накачкой и накачкой в противоположном направлении: РМ SMF - одномодовое волокно с сохранением поляризации, SMF - одномодовое волокно, EDF - активное волокно, WDM - спектральноселективный ответвитель, DCF - волокно со смещенной дисперсией, PBS - поляризационный делитель пучка, LD - лазерный диод 
В эксперименте были опробованы схемы с различными направлениями накачки (вставка на рис. 1). В каждой конфигурации проводился поиск импульсного режима при различной ширине волоконного фильтра. Зная частоту повторения импульсов в лазере и измеряя среднюю мощность, можно получить оценку для энергии импульса. Измерение средней мощности выходного излучения проводилось с использованием полупроводникового детектора S120C (Thorlabs, Inc.). Во всех экспериментах частота повторения лежала вблизи 3,3 МГц. При сонаправленной накачке получена генерация импульсов с энергией 3,3-3,7 нДж в широком диапазоне ширин фильтра, на рис. 2, $a$ приведены спектры импульсов для конфигураций с шириной фильтра 30 и 42,5 нм. Приведенные спектры по форме соответствуют характерным спектрам СЧДС. Примечательно, что несмотря на существенное увеличение энергии импульсов и значительно больший коэффициент рамановского усиления, в оптическом спектре не наблюдается ВКР-генерации на смещенной (на 100 нм) стоксовой длине волны.

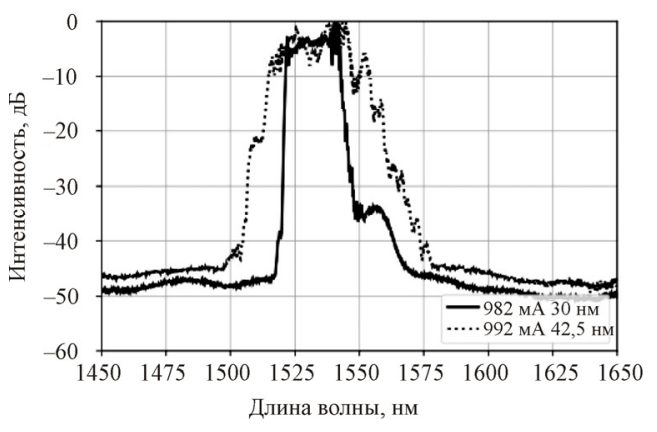

$a$

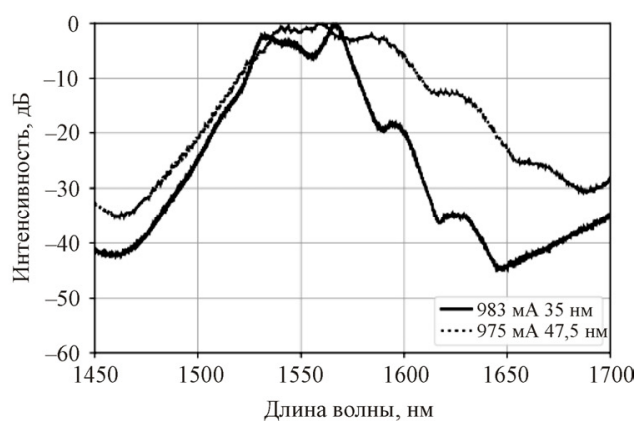

$\sigma$

Рис. 2. Оптические спектры генерируемых импульсов в различных конфигурациях: $a$ - сонаправленная накачка; $\sigma$ - накачка в противоположном направлении

Использование накачки в противоположном направлении привело к тому, что энергия импульса увеличилась до 5,1-5,9 нДж (рис. 2, б), а наблюдаемый спектр значительно уширился и потерял характерную для СЧДС форму с резкими краями. Полученный режим был охарактеризован более детально - проведено измерение оптического спектра, интерферометрической автокорреляционной функции и радиочастотного спектра (рис 3.) В статье [9] авторы приводят несколько режимов генерации, в том числе шумоподобные импульсы (noise-like pulses) - 
импульсы, структура АКФ которых содержит узкий пик ( 100 фс) на широком пьедестале (>10 пс). Полученные данные (рис.3) качественно демонстрируют наличие как пьедестала, так и фемтосекундного пика. Таким образом, можно сделать вывод, что генерируемые в схеме со встречной накачкой импульсы являются шумоподобными.

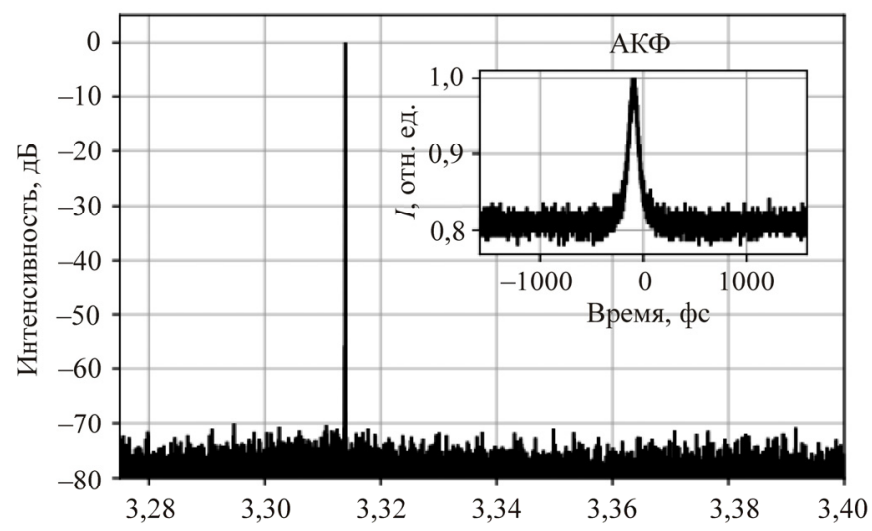

Рис. 3. Радиочастотный спектр генерации шумоподобных импульсов на фундаментальной частоте повторений (вставка: АКФ получаемых импульсов)

В ходе работы было продемонстрировано существенное увеличение энергии импульсов в задающем полностью волоконном эрбиевом генераторе с составным кольцевым резонатором. Использование волокна с сохранением поляризации и нормальной дисперсией позволило снизить частоту повторения до 3,3 МГц без потери режима генерации СЧДС. Максимальная энергия импульсов на выходе достигла 3,7 нДж при длине волны генерации 1530 нм (центр спектра импульса). Использование встречной схемы накачки привело к генерации шумоподоных импульсов с энергией до 5,9 нДж. Важно отметить, что существенное увеличение энергии импульсов не привело к ВКР генерации в длинноволновой области.

Работа выполнена при финансовой поддержке темы госзадания ИАиЭ СО РАН №0319-2018-0004 (работа Д.С. Харченко) и проекта № 38 (0319-2017-0011) комплексной программы фундаментальных научных исследований СО РАН «Междисииплинарные интеграционные исследования» (работа И.С. Жданова и С.А. Бабина). 


\section{Список литературы}

1. Stimulated Raman scattering microscopy with a robust fibre laser source / C.W. Freudiger, W. Yang, G.R. Holtom, N. Peyghambarian, X.S. Xie, K.Q. Kieu // Nat. Photonics. - 2014. - № 8. - P. 153-159.

2. Synthesis of a single cycle of light with compact erbium-doped fibre technology / G. Krauss, S. Lohss, T. Hanke, A. Sell, S. Eggert, R. Huber, A. Leitenstorfer // Nat. Photonics. - 2010. - № 4. - P. 33-36.

3. Development of a prototype compact fibre frequency synthesiser for mobile femtosecond optical clocks / V.S. Pivtsov, B.N. Nyushkov, I.I. Korel, N.A. Koliada, S.A. Farnosov, V.I. Denisov // Quantum Electronics. - 2014. - Vol. 44. - № 6.

4. Newbury N.R., Swann W.C. Low-noise fiber-laser frequency combs // J. Opt. Soc. Am. B. - 2007. - Vol. 24, № 8. - P. 1756-1770.

5. Schneider A., Stillhart M., Günter P. High efficiency generation and detection of terahertz pulses using laser pulses at telecommunication wavelengths // Opt. Express. - 2006. - № 14. - 5376.

6. $20 \mathrm{~nJ} 200 \mathrm{fs}$ all-fiber highly chirped dissipative soliton oscillator / D.S. Kharenko, E.V. Podivilov, A.A. Apolonski, S.A. Babin // Opt. Lett. 2012. - Vol. 37, № 19. - P. 4104-4106.

7. All-fiber highly chirped dissipative soliton generation in the telecom range / D.S. Kharenko, I.S. Zhdanov, A.E. Bednyakova, E.V. Podivilov, M.P. Fedoruk, A. Apolonski, S.K. Turitsyn, S.A. Babin // Opt. Letters. - 2017. - Vol. 42, № 16. - P. 3221-3224.

8. Özgören K., Ilday F.Ö. All-fiber all-normal dispersion laser with a fiber-based Lyot filter // Opt. Lett. - 2010. - Vol. 35, № 8. - P. 1296-1298.

9. Mode-locked fiber lasers with significant variability of generation regimes / S. Kobtsev, S. Smirnov, S. Kukarin, S. Turitsyn // Opt. Fib. Tech. 2014. - Vol. 20, № 6. - P. 615-620.

\section{References}

1. Freudiger C.W., Yang W., Holtom G.R., Peyghambarian N., Xie X.S., Kieu K.Q. Stimulated Raman scattering microscopy with a robust fibre laser source. Nat. Photonics., 2014, no. 8, pp. 153-159.

2. Krauss G., Lohss S., Hanke T., Sell A., Eggert S., Huber R., Leitenstorfer A. Synthesis of a single cycle of light with compact erbiumdoped fibre technology. Nat. Photonics., 2010, no. 4, pp. 33-36. 
3. Pivtsov V.S., Nyushkov B.N., Korel I.I., Koliada N.A., Farnosov S.A., Denisov V.I. Development of a prototype compact fibre frequency synthesiser for mobile femtosecond optical clocks. Quantum Electronics, 2014, vol. 44, no. 06.

4. Newbury N.R., Swann W.C. Low-noise fiber-laser frequency combs. J. Opt. Soc. Am. B, 2007, vol. 24, no. 8, pp. 1756-1770.

5. Schneider A., Stillhart M., Günter P. High efficiency generation and detection of terahertz pulses using laser pulses at telecommunication wavelengths. Opt. Express., 2006, no. 14, 5376.

6. Kharenko D.S., Podivilov E.V., Apolonski A.A., Babin S.A. $20 \mathrm{~nJ}$ 200 fs all-fiber highly chirped dissipative soliton oscillator. Opt. Lett., 2012, vol. 37, no. 19, pp. 4104-4106.

7. Kharenko D.S., Zhdanov I.S., Bednyakova A.E., Podivilov E.V., Fedoruk M.P., Apolonski A., Turitsyn S.K., Babin S.A. All-fiber highly chirped dissipative soliton generation in the telecom range. Opt. Letters., 2017, vol. 42, no. 16, pp. 3221-3224.

8. Özgören K., Ilday F.Ö. All-fiber all-normal dispersion laser with a fiber-based Lyot filter. Opt. Lett., 2010, vol. 35, no. 8, pp. 1296-1298.

9. Kobtsev S., Smirnov S., Kukarin S., Turitsyn S. Mode-locked fiber lasers with significant variability of generation regimes. Opt. Fib. Tech., 2014, vol. 20, no. 6, pp. 615-620.

Получено 16.09.2018 